\title{
Protecting Embryo with Chinese Herbal Medicine for Treating Habitual Miscarriages----- 4 Cases Study Enclosed
}

\author{
Ms Dan Jiang* \\ Hallam Institute of TCM, UK \\ *Corresponding author: Ms Dan Jiang, Hallam Institute of TCM, 439 Glossop Road Sheffield S10 2PR, UK.

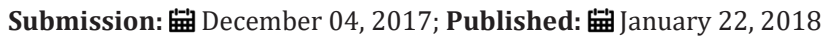

\section{Case Report}

It is almost impossible to protect the embryo, ladies successfully complete pregnancy, and have healthy babies, if they are going through vaginal bleeding, abdominal cramp and showing some other signs at the early month of pregnancy, which will normally end in miscarriages before TCM enters the West. Since the beginning of my practice of TCM (Acupuncture and Chinese herbal medicine) in UK, I have been using TCM for protecting embryo and terminating signs of miscarriage effectively in order to help ladies naturally complete their pregnancies and get healthy babies and there are more and more successful cases. I will share a few of the cases and my experience in treating miscarriage with Chinese herbal medicine and acupuncture as below:

\section{Case 1}

After 3 times of spontaneous abortions, her embryo was successfully protected with TCM treatment at the early time of pregnancy and she labored a healthy baby boy: Ms. S J, 38 years old of IT technician, started her menstrual bleeding at 4 weeks after her last period, but she already found a positive result after pregnancy test. She recognized that she might have a miscarriage again, because she has experienced these vaginal bleeding for three times in two years, all of which resulted in abortion in 7-8 weeks of her pregnancies after a gradually increased vaginal bleeding. She started her bleeding early this time, so she urgently went to see her GP requiring an Ultrasound to prove her pregnancy and a suitable treatment. She was refused an Ultrasound due to the reason that a regular one should be done at the 8th week of pregnancy and not any available treatment was given. When she visited me in anxiety, I checked that she had a vaginal bleeding in average amount with fresh blood, without clotting, or any solid substances in the blood, and abdominal cramp for 2-3 days. Last period of hers was 34 days ago, and the bleeding started 2 days ago without overreaction symptoms of pregnancy. Pregnancy test was positive that morning. Light red tongue with less white coating; Rolling and fine pulse. Diagnosis of Conventional western medicine:
A. The early signs of miscarriage
B. The $4^{\text {th }}$ of weeks of pregnancy
C. Differentiation of syndrome by TCM: deficiency of both spleen and Kidney
D. Weak embryo
E. Treating principle: Reinforcing kidney and spleen, Strengthening embryo

F. Herbal medical prescription: Shudihuang (Radix Rehmanniae Praeparata) 10, Huangqi (Radix Astragali)15, Baizhu (RhizomaAtractylodis Macrocephalae) 10, Aiye(Folium Artemisiae Argyi) 10 Sangjisheng (Herba Taxilli) 10, Xuduan (Radix Dipsaci) 10, Sharen(Fructus Amomi) 10, Ejiao (Colla Corii Asini) 10, Huangqin (Radix Scutellariae) 10, Chaihu (Radix Bupleuri) 10, Dangshen (Radix Codonopsis Pilosulae) 10, Fuling (Poria )10, Zhigancao (Radix Glycyrrhizae Praeparata) 5

Above herbal prescription was decocted into herbal juice, and she drank the juice, twice daily.

Result: After she drank the herbal juice for two days, her vaginal bleeding started to reduce until it completely stopped in two weeks; her abdominal cramp eased after her taking this herbal juice for a month. She was given an ultrasound in the $8^{\text {th }}$ week of her pregnancy by her GP which reported that she was in a normal pregnancy procedure. She remained well with a strong rolling pulse and without abnormal symptoms until $12^{\text {th }}$ weeks of her pregnancy; she went forward in her normal procedure after I ended the herbal medicine and labored a healthy baby boy in the full term.

\section{Case 2}

Due to 3 times of spontaneous abortions caused by high prolactin, she labored a baby girl after acupuncture and herbal medicine treatment: Mrs. C F, 33 years old of secretory, suffered from vaginal bleeding at the $8^{\text {th }}$ weeks in the third of her pregnancies, and she was terminated again with drug due to occurring spontaneous 
vaginal bleeding. Her gynecological consultant did all of relevant examinations for her, and a high prolactin was confirmed to be the reason for her multiple spontaneous abortions. Due to the many emotional symptoms she was suffering from, she was referred to see me---a TCM practitioner by her gynecological consultant.

Current figures: she was weepy with continuous tears and couldn't control her moodiness, bad depression, abdominal cramp. Due to the drug she was taking for cleaning her uterus the day before, she still had a bit of spotty blood, headache, insomnia, anxiety, loose bowel and fatigues. Red tongue, worse at tip and sides with less thin coating; Wiry-fine pulse. Prolactin 901miu/I, repeat prolactin $839 \mathrm{miu} / \mathrm{I}$ Rest of hormonal test: normal. Diagnosis of conventional western medicine:

\section{A. Habitual Miscarriage}

B. High prolactin

C. Differentiation of syndrome by TCM: Liver Qi stagnation and spleen deficiency

D. Liver fire flaring up

E. Acupuncture treatment: Du20 (Baihui), Du24 (Shenting), St8 (Touwei, Emotion area in Scalp acupuncture), Sj5 (Waiguan) Gb41 (Zulinqi), Li4 (Hegu), Liv3 (Taichong), Ren3 (Zhongji), Ren6 (Qihai), Ext (Zigong), Sp9 (Yinlingquan)

F. Chinese herbal medicine: The first step: to release the accumulated blood from the uterus, to expel stagnated liver Qi and to clear excessive Liver heat.

G. Herbal prescription: Chaihu (Radix Bupleuri) 10, Zhiqiao (FructusAurantii) 10, Chishao (Radix Paeoniae Rubra) 10, Ezhu (Rhizoma Curcumae) 10,Yimucao (Herba Leonuri) 10, Taoren (Semen Persicae) 10,Honghua (FlosCarthami) 10, Huanglian (Rhizoma Coptidis) 6, Zhizi (Fructus Gardeniae) 10, Suanzaoren (Semen Ziziphi Spinosae) 10, Fushen (Poria) 10, Chuanxiong (Radix Chuanxiong) 10

After she took the above herbal decocted juice, twice daily for a week, she recovered from last miscarriage: all of the bleeding stopped, her emotion calmed down, she had better sleep quality. The secondary step: to release the stagnated Liver Qi and to strengthen the spleen; to regulate menstrual circles, and to promote conception. Same acupuncture treatment once per week and patent herbal medicine: Chaihushugan Pill [1] and Renshenguipi Pill [2] were taken in the first two weeks of menstrual circles; Xiaoyao Pill [3] and Jinkuishenqi Pills [4] were taken in the last two weeks of menstrual circles.

Result: she had a normal prolactin after she took above treatment for three months; her natural pregnancy happened again in another month, and she started worry again as soon as she knew her pregnancy. She had minor symptoms of pregnancy reaction, nausea, vomiting, and minor vaginal bleeding, and she was given patent herbal Jiaweixiaoyao Pills [5] and Jinkuishenqi Pills [4] for stabilizing her emotion and easing vaginal bleeding until the $12^{\text {th }}$ weeks of her pregnancy. She went forward into a normal pregnancy procedure after $12^{\text {th }}$ week and naturally labored a healthy baby girl in the full term (Figure 1).

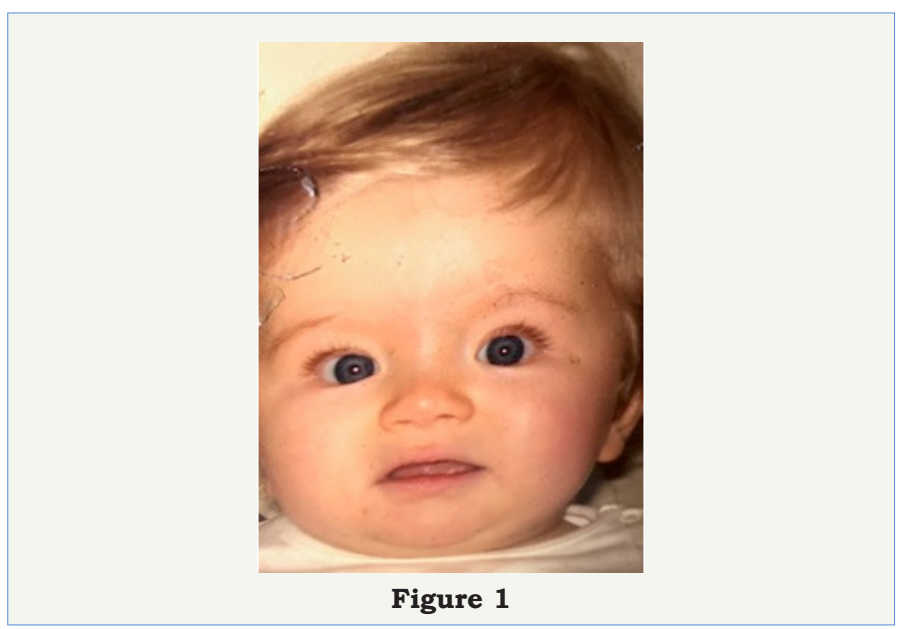

\section{Case 3}

Lady with Immune infertility met vaginal bleeding in pregnancy, but labored a baby boy from protected embryo by TCM treatment: Mrs. CC, a 27 year old spot trainer, couldn't conceive after living with her partner for 8 years. She was given a diagnosis of immune infertility, which means she possessed the positive antibody in her cervix which was against the sperm of her partner when she visited Infertility expert. They were told IVF was the only available way to result in pregnancy for them. They were in the waiting list for their IVF, and during this time she came to me and wanted to try TCM to see whether it can supply a miracle result. Current figures: quite fit as a spot trainer with regular menstrual circles, only minor depression. Light red tongue with thin white coating and wiry pulse. Diagnosis of Conventional western medicine: Immune Infertility Differentiation of syndrome by TCM: Liver Qi stagnation and Spleen deficiency.

\section{Treatment}

A. Acupuncture: Du20 (Baihui), Gb20 (Fengchi), Ren4 (Guanyuan), Ren6 (Qihai) Sp9 (Yinlingquan), Sp6 (Sanyinjiao), St 36 (Zusanli), Sj5 (Waiguan), Gb41 (Zulinqi)

B. Patent herbal medicine: Jiaweixiaoyao pills [5] and Buzhongyiqi Pills [6].

Above acupuncture was given once per week for a month, then once for two weeks; Patent herbs were taken twice daily. Mr. AC, 32 years old of IT technician, who was found lower activity in his sperm, was give Chinese herbal medicine for promoting the quality of his sperm: Jiaweixiaoyao Pills [5] and Wuziyanzong Pills [7].

Result: when Mrs. C gotpregnant naturally, this couple was very excited, but she experienced a vaginal bleeding when she was the 6th week of pregnancy. Due to light spotty blood and some pregnancy symptoms she suffered, I had to continue my treatment for miscarriage prevention.

A regular acupuncture and patent herbal medicine: Xiangshayangwei Pills [8] and Jinkuishenqi Pills [4] were given to 
her until the $12^{\text {th }}$ of weeks of her pregnancy. She labored a baby girl in the full term (Figure 2).

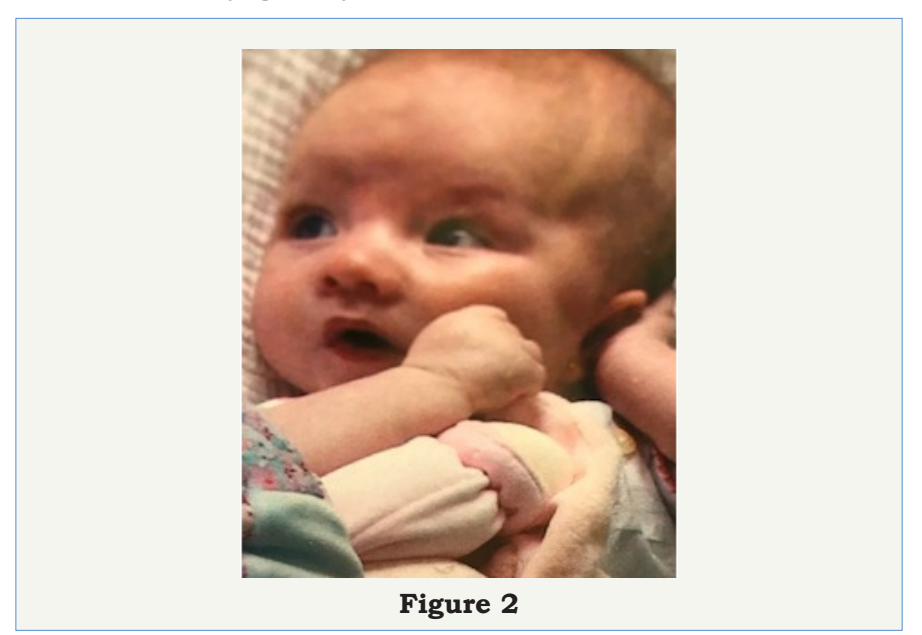

\section{Case 4}

The lady with Infertility caused by Erythema Lupus, was pregnant and her embryo protected, pregnancy procedure managed by TCM until her having a healthy baby by a caesarean:

Mrs. R B, 38 years old of officer, suffered from active Erythema Lupus for 10 years and kept taking oral steroid treatment which couldn't be stopped, otherwise she might experience recurring palpitation and aching in general joints. She was staying in the stable state under the control of $10 \mathrm{~g}$ Progesterone daily. Since her immune system were staying in an active state as a result of the medication, she couldn't get pregnant: she lived with her stalwart husband for near 10 years of expecting their baby, but no positive result. Due to their expectation, she was gradually reducing her steroid dose and was referred to me for accepting a TCM treatment, and observed by Rheumatoid-immune and Gynecological consultants. Current figures: because she had just stopped the Progesterone, she felt aching in general joints, fatigue, anxiety, and she was also nervous and restless; she had regular menstrual circles with a normal blood amount and no pain, but many symptoms of pre-menstrual tension; loose bowel 2-3 times daily, disturbed sleep. Red tongue without coating, Wiry-fine pulse. Diagnosis of Conventional western medicine:

a) Erythema Lupus (in activity)

b) Infertility

c) Differentiation of syndromes by TCM: Liver Qi stagnation and Spleen deficiency

d) Dampness accumulated throughout three burners

\section{Treatment}

Acupuncture: Moxi: Ren8 (Shenque) Du20 (Baihui), Gb20 (Fengchi), Li11 (Quchi), Lu7 (Lieque), Sp10 (Xuehai), Sp9 (Yinlingquan), Sp6 (Sanyinjiao), Ki3 (Taixi), Ki2 (Xingjian), Ki6 (Zhaohai)

Among of them: Li11 and Sp10 were given special handle technique for expelling excessive dampness throughout three burners. In the first step of her herbal treatment:

a) Treating principle: to release excessive dampness from meridians and collateral channels; to expel out accumulated Liver Qi and to reinforce spleen.

b) Chinese herbal medicine in the concentrated herbal powder form is prescribed as below: Qianghuo (Radix EtRhizoma Notopterygii) 15, Duhuo (Radix Angelicae Pubescentis) 10, Rendongteng (Caulis Lonicerae) 15, Fuling (Poria) 10, Chaihu(Radix Bupleuri) 10, Baizhu (RhizomaAtractylodisMacrocephalae) 10, Dangui (Radix AngelicaeSinensis) 10, Chuanxiong (Radix Chuanxiong) 10, Chishao (Radix PaeoniaeRubra) 10, Zhishi (FructusAurantiiImmaturus) 10, Jixueteng (Caulis Millettiae; Caulis Spatholobi) 20, Zhigancao (Radix GlycyrrhizaePraeparata) 5.

Above acupuncture was given once per week, herbal powders were taken twice daily for two months, as a result she had no joint pain, and she had regular menstrual circle, calm mood and balance in general body. In the second step of her treatment: Treating principle: to regulate the menstrual circles and to promote pregnancy. Acupuncture was similar to the last step and herbal powders were prescribed as below: Shengdihuang (Radix Rehmanniae) 30, Mudanpi (Cortex Moutan) 10, Shanzhuyu(Fructus Corni) 10, Shanyao (Rhizoma Dioscoreae) 10, Baizhu(Rhizoma Atractylodis Macrocephalae) 10, Zexie (Rhizoma Alismatis) 10, Fuling (Poria) 10, Rendongteng(Caulis Lonicerae) 10, Yimucao (HerbaLeonuri) 10, Chishao (Radix PaeoniaeRubra) 10, Chuanxiong (Radix Chuanxiong;) 10, Zelan (Herba Lycopi) 10, Xiangfu (Rhizoma Cyperi) 10, Taoren (Semen Persicae) 10, Honghua(Flos Carthami) 10 , were added in prescription in the secondary week; Xianmao (Rhizoma Curculiginis) 10, Yinyanghuo (Herba Epimedii) 15 and Sharen(Fructus Amomi) 10 were added in prescription from $3^{\text {rd }}$ to $4^{\text {th }}$ week.

After a regular treatment for two months, Mrs. R B disappeared and stopped coming for treatment. In the third step for her treatment: When she came back after four months, I was told that she was pregnant, and she believed she had a successful pregnancy, so stopped accepting her TCM treatment as soon as she was pregnant, but she experienced miscarriage after vaginal bleeding for a few days when she was 8th weeks in pregnancy. After three months of rest, she came again for another attempt.

After TCM treatment of strengthening spleen and kidney, regulating menstrual circles and promoting pregnancy for two months, she was pregnant again. We had to continue TCM treatment during her pregnancy and monitor her Erythema Lupus and immune system. Her prescription of herbal powder was as below: Shudihuang(Radix Rehmanniae Praeparata) 30, Huangqi (Radix Astragali) 30, Sangjisheng (Herba Taxilli) 10, Xuduan (Radix Dipsaci) 10, Baizhu (Rhizoma Atractylodis Macrocephalae) 10, Gouqizi (Fructus Lycii) 10, Huangqin(Radix Scutellariae) 10, Sharen(FructusAmomi) 10, Ejiao (Colla Corii Asini) 10, Banxia(Rhizoma Pinelliae ) 10, Chenpi(Pericarpium Citri Reticulatae) 10, Fuling(Poria) 10, Zhigancao (Radix Glycyrrhizae Praeparata) 5. 
I made a bit of modification each time according to her state until $12^{\text {th }}$ weeks, and she still manifested some abdominal cramp, general joint pains, unstable ESR levels, fatigue, anxiety, nausea and relevant symptoms more or less sometimes, so I adjusted the herbs to match her symptoms. I was giving her TCM treatment under the observation of both Rheumatoid-immune and Gynecological consultants, but they only observed to guarantee that she was staying in a reasonable condition. She was given a caesarean

Table 1: Ingredients of the patent herbal formulae in paper. when she experienced an increasing ESR in the $32^{\text {th }}$ of weeks which resulted in a possible aggravation of her immune system; Gynecological consultant thought the baby was big enough for a healthy life, so a caesarean was conducted. After terminating her pregnancy, her immune system gradually recovered, and she never used steroid anymore and remained in a good state. Both of the consultants appreciated the effective complementary effects of TCM (Table 1).

\begin{tabular}{|c|c|c|c|c|}
\hline No & $\begin{array}{l}\text { Names of patent herbal } \\
\text { formulae }\end{array}$ & $\begin{array}{l}\text { Ingredients of formulae in } \\
\text { Chinese spelling names }\end{array}$ & Ingredients of formulae in Latin name & Dose \\
\hline \multirow{20}{*}{1} & \multirow{20}{*}{ Chaihushugan Pills } & Fuling & Poria & 12.6 \\
\hline & & Zhiqiao & FructusAurantii & 10 \\
\hline & & Baidoukou & Fructus Amomi Cardamomi & 9 \\
\hline & & Baishao & Paeonia Lactiflora Pall & 5.4 \\
\hline & & Xiangfu & RhizomaCyperi & 3.6 \\
\hline & & Chenpi & Pericarpium Citri Reticulatae & 3.6 \\
\hline & & Jiegeng & Radix Platycodi & 3.6 \\
\hline & & Shanzha & Fructus Crataegi & 3.6 \\
\hline & & Shenqu & Massa Fermentata Medicinalis & 3.6 \\
\hline & & Chaihu & Radix Bupleuri & 3.6 \\
\hline & & Huangqin & Radix Scutellariae & 3.6 \\
\hline & & Bohe & Herba Menthae & 3.6 \\
\hline & & Zisugeng & Caulis Perillae & 3.6 \\
\hline & & Sanleng & Rhizoma Sparganii & 3.6 \\
\hline & & Dahuang & Radix Et Rhizoma Rhei & 3.6 \\
\hline & & Qingpi & Pericarpium Citri Reticulatae Viride & 3.6 \\
\hline & & Danggui & Radix Angelicae Sinensis & 3.6 \\
\hline & & Ezhu & RhizomaCurcumae & 3.6 \\
\hline & & Fangfeng & Radix Saposhnikoviae Divaricatae & 9 \\
\hline & & Gancao & Radix Glycyrrhizae & 3.6 \\
\hline \multirow{10}{*}{2} & \multirow{10}{*}{ Renshenguipi Pills } & Renshen & Radix Ginseng & 15.25 \\
\hline & & Baizhu & Rhizoma Atractylodis Macrocephalae & 10.17 \\
\hline & & Huangqi & Radix Astragali & 10.17 \\
\hline & & Fushen & Poria & 10.17 \\
\hline & & Yuanzhi & Radix Polygalae & 10.17 \\
\hline & & Suanzaoren & Semen Ziziphi Spinosae & 10.17 \\
\hline & & Longyanrou & Arillus Longan & 10.17 \\
\hline & & Danggui & Radix Angelicae Sinensis & 10.17 \\
\hline & & Dazao & Fructus Zizyphi Jujubae & 10.17 \\
\hline & & Zhigancao & Radix Glycyrrhizae & 3.39 \\
\hline \multirow{7}{*}{3} & \multirow{7}{*}{ Xiaoyao Pills } & Chaihu & Radix Bupleuri & 16.67 \\
\hline & & Dangui & Radix AngelicaeSinensis & 16.67 \\
\hline & & Baishao & Radix Paeoniae Alba & 16.67 \\
\hline & & Baizhu & Rhizoma Atractylodis Macrocephalae & 16.67 \\
\hline & & Fuling & Poria & 16.67 \\
\hline & & Bohe & HerbaMenthae & 3.32 \\
\hline & & Zhigancao & Radix Glycyrrhizae & 13.33 \\
\hline
\end{tabular}




\begin{tabular}{|c|c|c|c|c|}
\hline \multirow{10}{*}{4} & \multirow{10}{*}{ Jinkuishenqi Pills } & Shudihuang & Rehmannia Glutinosa, Libosch & 28.1 \\
\hline & & Shanyao & Dioscorea OppositaThunb & 7.1 \\
\hline & & Shanzhuyu & Fructus Corni & 7.1 \\
\hline & & Mudanpi & Cortex Moutan & 7.1 \\
\hline & & Zexie & Rhizoma Alismatis & 7.1 \\
\hline & & Fuling & Poria & 21 \\
\hline & & Guizhi & Ramulus Cinnamomi & 7.1 \\
\hline & & Rougui & Cortex Cinnamomi & 1.2 \\
\hline & & Niuxi & Radix Achyranthis Bidentatae & 7.1 \\
\hline & & Cheqianzi & Semen Plantaginis & 7.1 \\
\hline \multirow{9}{*}{5} & \multirow{9}{*}{ Jiaweixiaoyao Pills } & Chaihu & Bupleurum Chinese DC & 11 \\
\hline & & Dangui & Radix AngelicaeSinensis & 11 \\
\hline & & Baishao & Paeonia Lactiflora Pall & 11 \\
\hline & & Baizhu & Rhizoma Atractylodis Macrocephalae & 11 \\
\hline & & Fuling & Poria & 11 \\
\hline & & Mudanpi & Cortex Moutan & 17 \\
\hline & & Zhizi & Gardenia Jasminoides Sllis & 17 \\
\hline & & Bohe & Herba Menthae & 2 \\
\hline & & Gancao & Glycyrrhizae Uralensis Fisch. & 9 \\
\hline \multirow{8}{*}{6} & \multirow{8}{*}{ Buzhongyiqi Pills } & Huangqi & Radix Astragali & 30 \\
\hline & & Dangshen & Radix Codonopsis Pilosulae & 10 \\
\hline & & Baizhu & Altractylodes Macrocephala Koidz & 9 \\
\hline & & Danggui & Radix Angelicae Sinensis & 9 \\
\hline & & Shengma & Rhizoma Cimicifugae & 9 \\
\hline & & Chaihu & Bupleurum Chinese DC & 9 \\
\hline & & Chenpi & Pericarpium Citri Reticulatae & 9 \\
\hline & & Zhigancao & Glycyrrhizae Uralensis Fisch. & 15 \\
\hline \multirow{5}{*}{7} & \multirow{5}{*}{ Wuziyanzong Pills } & Gouqizi & Fructus Lycii & 34.78 \\
\hline & & Tusizi & Semen Cuseutae; Semen Cuseutae & 34.78 \\
\hline & & Fupenzi & Fructus Rubi & 17.39 \\
\hline & & Wuweizi & Fructus Schisandrae & 4.35 \\
\hline & & Chqianzi & Herba Plantaginis & 8.7 \\
\hline \multirow{12}{*}{8} & \multirow{12}{*}{ Xiangshayangwei Pills } & Juye & Folium CitriTangerinae & 7.6 \\
\hline & & Sharen & FructusAmomi & 7.6 \\
\hline & & Baizhu & Rhizoma Atractylodis Macrocephalae & 10.87 \\
\hline & & Chenpi & Pericarpium Citri Reticulatae & 10.87 \\
\hline & & Fuling & Poria & 10.87 \\
\hline & & Banxia & Rhizoma Pinelliae & 10.87 \\
\hline & & Xiangfu & Rhizoma Cyperi & 7.6 \\
\hline & & Zhishi & Fructus AurantiiImmaturus & 7.6 \\
\hline & & Doukou & Fructus Amomi Card amomi & 7.6 \\
\hline & & Houpo & Cortex Magnoliae Officinalis & 7.6 \\
\hline & & Huoxiang & Herba Agastaches & 7.6 \\
\hline & & Gancao & Radix Glycyrrhizae & 3.32 \\
\hline
\end{tabular}




\section{Analysis}

There are accurate effects from traditional Chinese herbal medicine for protection of embryo and treatment of miscarriage as observed from above cases. We can explore the advantages and shortcomings of TCM treatment from these cases:

A. For those spontaneous miscarriages for which no causes are found by conventional western medicine, they can occur from preclinical state. TCM believe that might be a result of Qi and blood deficiency. For example, the lady might be pregnant when she is in a weaker body state; or reconceive in the short time after last miscarriage; or does not have enough rest for complete recovery from a disease...... due to above conditions, cervix may be too loose to hold the embryo, and after a bit of movement, the placenta and embryo can be released to cause a miscarriage. TCM will play an effective role in helping with these conditions, like in case 1.

B. For ladies' miscarriage caused by emotional or psychological disorders, or high prolactin, which is always accompanied with psychological symptoms, or immune disorder, TCM can offer an effective treatment. Like case2, 3 and 4.

C. The ladies who became pregnant assisted from TCM promotion or IVF support may easily experience miscarriages, especially for the cases when the ladies conceive very quickly, since the Qi and blood deficiency of these ladies have not been reinforced enough, so we should continue TCM treatment in order to prevent miscarriage's occurrence and help ladies go forward in their pregnancy peacefully.

D. For the ladies with miscarriage related with Gene disorder or abnormal Chromosome, TCM cannot present a satisfactory effect. This may need a longer time of observation and treatment to sort out.

\section{References}

1. Li X, Zhang M, Wang B, Li Y, Wang L, et al. (2013) Shenghua Decoction reduces uterine bleeding and regulates T-cell paradigm in human deciduas of RU486 medical abortion. J Ethnopharmacol 150(3): 907917.

2. Hullender Rubin L, Cantor Dand Marx BL (2013) Recurrent Pregnancy Loss and Traditional Chinese Medicine. Med Acupunct 25(3): 232-237.

3. Gao J and Luo SP (2011) Kidney-tonifying and abortion preventing effects of Shou Tai Wan by different extracted methods on rats. Zhong Yao Cai 34(8): 1251-1255.

4. Sun Fand, Yu J (1999) Effect of TCM on plasma beta-endorphin and placental endocrine in threatened abortion. Zhongguo Zhong Xi Yi Jie He Za Zhi 19(2): 87-89.

5. Wang QM, Yao DY, Yang YF (1994) Experimental study on anti-abortion effect of Yunkang oral liquid. Zhongguo Zhong Xi Yi Jie He Za Zhi 14(7): 418-420.

6. Zhao LQ (2013) The treatment of recurrent spontaneous miscarriage. Medical condition overview. The Journal of Chinese Medicine 16(101): 13.

7. Dan Jiang, Lily Li, Bai-Yun Zeng (2017) Treatment of Chinese Herbal Medicine for Female Infertility. In: Bai-Yun Zeng, Kaicun Zhao (Eds.), International Review of Neurobiology. Academic Press, Burlington, USA, Vol 135, pp. 233-247.

8. Dan Jiang, Alberto Coscione, Lily Li, Bai-Yun Zeng (2017) Effect of Chinese Herbal Medicine on Male Infertility. In: Bai-Yun Zeng, Kaicun Zhao (Eds.), International Review of Neurobiology. Academic Press, Burlington, USA, Vol 135, pp. 297-311. 